Draft Version June 2, 2018

Preprint typeset using LATEX style emulateapj v. 08/22/09

\title{
THE FATE OF HIGH-VELOCITY CLOUDS: WARM OR COLD COSMIC RAIN?
}

\author{
Fabian Heitsch ${ }^{1}$ and Mary E. Putman ${ }^{1,2}$ \\ Draft version June 2, 2018
}

\begin{abstract}
We present two sets of grid-based hydrodynamical simulations of high-velocity clouds (HVCs) traveling through the diffuse, hot Galactic halo. These HI clouds have been suggested to provide fuel for ongoing star formation in the Galactic disk. The first set of models is best described as a wind-tunnel experiment in which the HVC is exposed to a wind of constant density and velocity. In the second set of models we follow the trajectory of the HVC on its way through an isothermal hydrostatic halo towards the disk. Thus, we cover the two extremes of possible HVC trajectories. The resulting cloud morphologies exhibit a pronounced head-tail structure, with a leading dense cold core and a warm diffuse tail. Morphologies and velocity differences between head and tail are consistent with observations. For typical cloud velocities and halo densities, clouds with HI masses $<10^{4.5} \mathrm{M}_{\odot}$ will lose their HI content within $10 \mathrm{kpc}$ or less. Their remnants may contribute to a population of warm ionized gas clouds in the hot coronal gas, and they may eventually be integrated in the warm ionized Galactic disk. Some of the (still over-dense, but now slow) material might recool, forming intermediate or low velocity clouds close to the Galactic disk. Given our simulation parameters and the limitation set by numerical resolution, we argue that the derived disruption distances are strong upper limits.
\end{abstract}

Subject headings: Galaxy:halo — Galaxy:evolution — ISM:HI — hydrodynamics — turbulence — methods:numerical

\section{MOTIVATION}

Galaxy disks require gas to fuel their ongoing star formation, yet the fueling mechanism and the nature of the gas reservoir remain unclear. In galaxy formation models, the halo gas is initially heated as it follows the dark matter and subsequently cools and falls to the disk (e.g., White \& Rees 1978; White \& Frenk 1991). Even at $z=0$, halo gas is proposed to gradually condense out into clouds, and a significant amount $\left(\sim 4 \times 10^{10} \mathrm{M}_{\odot}\right)$ of hot gas is thought to remain in an extended $(\sim$ $150 \mathrm{kpc}$ ), hot, diffuse halo medium (Maller \& Bullock 2004; Fukugita \& Peebles 2006; Kaufmann et al. 2006; Sommer-Larsen 2006). Other sources of potential fuel for galaxy disks include gas stripped from satellites, and possibly some mixed-in galactic fountain gas. Understanding the properties of the diffuse hot halo and the clouds that fuel galaxy disks is a crucial step towards comprehending how galaxies form and evolve.

Observationally we know halo gas exists today around galaxies that is likely to fall towards the disk $\left(\mathrm{v}_{\text {qas }}<\right.$ $\mathrm{V}_{\text {esc }}$ ) (e.g., Fraternali et al. 2001; Putman et al. 2002; Thilker et al. 2004). The neutral hydrogen halo clouds found around the Milky Way are called high-velocity clouds (HVCs) and are presumably a future source of star formation fuel for our Galaxy. HVCs are moving through a more diffuse halo medium as evident from their structure (e.g., Brüns et al. 2000; Putman et al. 2003a; Peek et al. 2007), and from detections of high-velocity OVI absorption (Sembach et al. 2003). The clouds are disrupted by this interaction and form a "head-tail" structure, i.e. the head of the cloud is compressed around a cold core, and a warmer, diffuse tail extends behind the

\footnotetext{
${ }^{1}$ Dept. of Astronomy, University of Michigan, 500 Church St. Ann Arbor, MI 48109-1042, U.S.A

2 Dept. of Astronomy, Columbia University, 550 W 120th St., New York, NY 10027, U.S.A
}

cloud. The properties and lifetime of a given head-tail cloud depend on the density of the diffuse halo medium. Head-tail clouds therefore present the opportunity to study the lifetimes of halo clouds and the density of the diffuse Galactic halo, a medium that is difficult to detect directly.

In this paper we present three-dimensional grid simulations of HVCs interacting with a diffuse halo medium and compare the results to neutral hydrogen observations. We discuss two sets of numerical experiments, one best described as a wind-tunnel experiment in which the model cloud is exposed to a wind of constant density and velocity, and a second set following the model cloud on its way through an isothermal, hydrostatic halo towards the disk. All models include equilibrium heating and cooling. The characteristic timescales and pathlengths for disruption are assessed in the context of fueling the Galactic disk with the observed population of HI HVCs. Our main results are summarized in Figure 6, predicting the distances HVCs of a given mass are expected to survive their disruption at a given cloud velocity and background halo density.

\section{TECHNICAL ASPECTS}

\subsection{The Numerical Scheme}

Calculations were performed with Proteus, an unsplit, second-order accurate gas-kinetic scheme (Prendergast \& Xu 1993; Slyz \& Prendergast 1999; Xu 2001; Heitsch et al. 2008) based on the Bhatnagar-GrossKrook formalism (Bhatnagar et al. 1954). The scheme conserves mass, momentum and total energy to machine accuracy, and it allows the explicit control of viscosity and heat conduction, albeit at a Prandtl number of 1 (see $\mathrm{Xu}$ 2001). The dissipative constants are set such that they control the (very low) intrinsic numerical dissipation (see Slyz et al. 2002, $\S 3$ for a detailed description). 
The thermal physics are implemented as a modification of the total energy via additional heating and cooling terms. In the absence of heating and cooling, the gas is adiabatic with an exponent of $\gamma=5 / 3$. The heating and cooling terms are accumulated in a tabulated "cooling function" (i.e. energy density change rate $\dot{e}$ ), depending on density $n$ and temperature $T$ as $\dot{e}=n \Gamma-n^{2} \Lambda(T)$. The energy change is done iteratively, i.e. if the local cooling timescale is smaller than the current CourantFriedrich-Levy (CFL) timestep, we subcycle on the energy equation in that cell. This is generally the case in the cold dense gas. Heating and cooling are operating throughout the whole simulation volume. We derive the cooling function from the rates given by Wolfire et al. (1995a) for $T<10^{4} \mathrm{~K}$, while for higher temperatures we use the rates of Sutherland \& Dopita (1993). We assume a metallicity of $10 \%$ solar (Wakker \& van Woerden 1997; Wakker 2001).

The heating is due to a generic UV radiation field, which is set to $G_{0}=1.1 X(z)$, where $X(z)=1 /[1+$ $\left.(z / 8.53 \mathrm{kpc})^{2}\right]$ (Wolfire et al. 1995b) with $z=10 \mathrm{kpc}$ as a "typical" HVC distance. Although we do not know the actual distance to each of the head-tail clouds, we adopt $10 \mathrm{kpc}$ for most models based on the recent work constraining the distances to many HVCs (Thom et al. 2006; Wakker et al. 2008). Many of the head-tail clouds are in the vicinity of larger complexes, suggesting that they may have a similar distance. This will be described further in the paper presenting the population of HIPASS head-tail clouds (Putman et al., in preparation). The UV-field is kept constant during the evolution of the HVC. All heating and cooling processes are local, and we do not address radiative transfer effects. Thus, the clouds cannot self-shield, and possible effects of increased UV radiation once the cloud approaches the Galactic plane cannot be addressed.

\subsection{Setup and Parameters}

We ran two sets of models. Series $W$ are wind-tunnel experiments, while in series $\mathrm{H}$, the cloud falls vertically towards the disk's midplane, traversing an isothermal halo medium in hydrostatic equilibrium. In both cases, the clouds are initially at rest, located in the lower quarter of the simulation domain.

All model clouds have an exponential density profile (e.g. Burton et al. 2001, see Figure 1), convolved by a tanh shifted to the nominal cloud radius to make the cloud compact. We also experimented with uniform density clouds and found that they tend to disrupt even faster than the centrally peaked clouds, as expected. Wind-tunnel experiments have simulation domain sizes varying between $150^{2} \times 300$ and $500^{2} \times 1000 \mathrm{pc}^{3}$, while the free-fall experiments use box sizes of $500^{2} \times 2000 \mathrm{pc}^{3}$.

\subsubsection{Wind Tunnel Experiments}

For series $\mathrm{W}$, the background medium has a constant density $n_{h}$ and temperature $T_{0}$ such that the pressure is $100 \mathrm{~K} \mathrm{~cm}^{-3}$ (Wolfire et al. 1995b) for models with $n_{h} \leq 10^{-4} \mathrm{~cm}^{-3}$, and $300 \mathrm{~K} \mathrm{~cm}^{-3}$ for models with $n_{h}=3 \times 10^{-4} \mathrm{~cm}^{-3}$ in order to avoid the peak of the cooling curve at $T \approx 3 \times 10^{5} \mathrm{~K}$. The cloud is initially in thermal pressure equilibrium with its surroundings. To keep the cloud within the simulation domain as long as possible, we balance a constant gravitational acceleration $\mathrm{g} \equiv g \hat{z}$ against a "wind" entering the bottom of the domain. The wind speed is ramped up linearly at a rate of $13 \mathrm{~km} \mathrm{~s}^{-1} \mathrm{Myr}^{-1}$ until the desired "cloud" velocity $v_{0}$ is reached. The velocity $v_{0}$ sets the size of the gravitational acceleration $g$, via

$$
M_{c} g=\pi R_{c}^{2} C_{D} \rho_{h} v_{0}^{2} / 2
$$

with the cloud mass $M_{c}$, the drag coefficient $C_{D}=1.0$ and the halo density $\rho_{h} \equiv n_{h} m_{H}$. We note that while equation (1) implicitly assumes that the clouds reach a terminal velocity $v_{0}$, we use it only as a means to achieve an approximate balance between gravity and ram pressure. It is not clear whether HVCs generally reach their terminal velocities (Benjamin \& Danly 1997; Peek et al. 2007). Moreover, equation (11) assumes that the clouds do not disintegrate, leading to an over-estimate of the terminal velocity $v_{0}$. The lower $z$-boundary is defined by the "wind", while all other boundaries are left open.

\subsubsection{Free-fall Experiments}

For series H, we drop the HVC in a hydrostatic isothermal halo at $T_{0}=10^{6} \mathrm{~K}$. We use a gravitational acceleration of $g=10^{-8} \mathrm{~cm}^{2} \mathrm{~s}^{-2}$, corresponding to the fit between 1 and $10 \mathrm{kpc}$ provided by Benjamin \& Danly (1997), and we assume a halo density of $n_{h}=10^{-4} \mathrm{~cm}^{-3}$ at $z=10 \mathrm{kpc}$. This results in a density profile of

$$
n_{h}(z)=n_{0} \exp \left(-g m_{H} /\left(k_{B} T_{0}\right)\right)
$$

with a mid-plane density of $n_{0}=4 \times 10^{-3} \mathrm{~cm}^{-3}$. The fact that this is lower than the volume density of the ionized gas in the Galactic midplane by a factor of $5-10$ (see e.g. Ferrière 2001) is of no concern here, since our simple model cannot describe the density structure for $z \lesssim 2 \mathrm{kpc}$ correctly anyway. We will discuss in $\$ 4.2$ how any systematic underestimate of the background halo density affects the disruption of the clouds. To keep the cloud within the simulation domain, the grid is shifted by exactly one cell to lower $z$ once the center of the HI mass distribution has fallen at least by one cell. The new layer of cells at the bottom of the domain is set according to the exponential density profile determined by the above parameters. This prevents unnecessary and diffusive interpolation and it allows us to follow the cloud on its trip through the halo.

Apart from this "comoving" grid, the major difference to series $\mathrm{W}$ is that the halo pressure is no longer constant. This leads to a successive compression of the cloud and its fragments, until they drop below the resolution limit of the dynamical instabilities relevant for cloud disruption (Roulston \& Ahrens 1997; Korvcansky et al. 2000). This effect is amplified by the increasingly higher cooling rates. In the extreme case, the remaining cloud fragment can collapse to a thin "needle" in essential free-fall. To prevent such unphysical behavior, we regrid the simulation whenever the most massive fragment drops below the resolution limit. For such models, we simply reduce the domain size by one half, at the same number of cells, thus doubling the spatial resolution. Typically, one regridding step is necessary to follow the cloud evolution until disruption. It should be noted that even with the regridding the smallest, cold dense fragments are not resolved with respect to the hydrodynamical instabilities. Thus, their lifetimes tend to be over-estimated. 


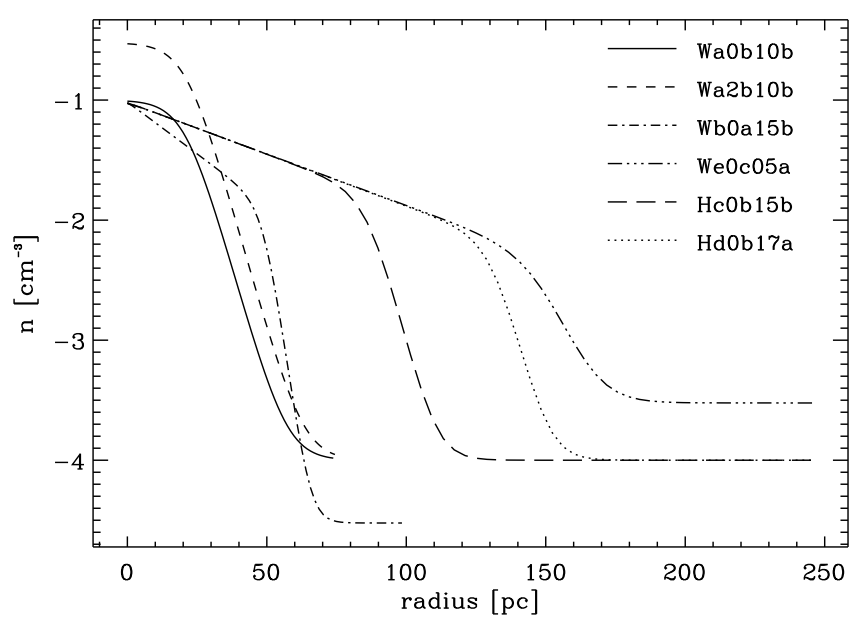

FIG. 1.- Logarithmic density profiles for models as indicated. The profiles are set by an exponential convolved with a tanh shifted to the nominal cloud radius to make the cloud compact.

\subsubsection{Model Names}

The model parameters are summarized in Table 1. The model names consist of seven characters, the first indicating whether the model is a wind-tunnel experiment $(\mathrm{W}$, \$2.2.1) or a free-fall experiment $(\mathrm{H}$, 2.2.2). The second character denotes the cloud radius $(a=21, b=50$, $c=89, d=133, e=150 \mathrm{pc}$ ). The initial peak cloud density is given by the third character in $0.1 \mathrm{~cm}^{-3}$, while the fourth character indicates the halo density $\left(a=3 \times 10^{-5}\right.$, $\left.b=1 \times 10^{-4}, c=3 \times 10^{-4} \mathrm{~cm}^{-3}\right)$. The fifth and sixth character stand for the nominal cloud velocity for windtunnel experiments, and for the peak cloud velocity for free-fall experiments, in $10 \mathrm{~km} \mathrm{~s}^{-1}$. Finally, the seventh character stands for the resolution along the shorter axes ( $a=128, b=256$ cells $)$.

\section{RESULTS}

We start our discussion of the results in terms of morphology (3.1), including a comparison to observed HVCs. A detailed view of three representative models is given in $\$ 3.2$. We also summarize the cloud life times and their travel distances (\$3.3), discuss resolution effects (3.4), and differences to previous work (3.5, see also $\longdiv { 4 . 2 . 2 }$.

\subsection{Morphologies}

Figure 2 shows column density maps of HI (i.e. gas at $T<10^{4} \mathrm{~K}$ for the model clouds) of observed and modeled HVCs with the same column density contours for each. Observed clouds were taken from the HI Parkes All-Sky Survey (HIPASS) cloud catalogue (Putman et al. 2002). Clouds with a head-tail structure were found for $\sim 40 \%$ of the small HVCs (CHVCs and :HVCs) in this catalogue (Putman et al., in prep.), and also along the Magellanic stream (Putman et al. 2003b). Brüns et al. (2000) put together a flux-limited sample of head-tail clouds with the Leiden-Dwingeloo Survey (35' resolution vs. $15.5^{\prime}$ for HIPASS) and found $20 \%$ of their entire sample show a velocity and column density gradient consistent with being head-tail clouds. The modeled clouds in Figure 2 have been degraded to a "beam width" of $3.0^{\prime}$, and they have been rotated by the amount indicated on top of each model map. For small rotation angles, the cloud is traveling nearly head-on towards the observer. Model Wb1a15b at $45^{\circ}$ develops a pronounced head-tail structure reminiscent of the structures in the observed cloud, including several fragments in the tail. Shear flow instabilities act along the cloud and tail flanks, and the tail itself shows kinks as a result of a turbulent wake. Cooling leads to fragmentation, resulting in "multiple cores" along the tail. In the head-on view (cloud Wb1a15b, $5^{\circ}$ ), the tail is nearly completely suppressed, but might be discernible in a velocity shift between the cold and warm gas component. We will explore the kinematic signatures of the clouds and their disruption in a subsequent paper. The panel on the right (model We1c05b, $30^{\circ}$ ) shows a more massive cloud at a late evolutionary stage. The cloud has disintegrated into multiple cores. The minimum temperatures in the cores reach $\approx 100 \mathrm{~K}$.

\subsection{Cloud Evolution and Disruption Mechanisms}

We will discuss the cloud evolution in terms of three models, namely Wb1a15b, Hc1b13a and (at twice the resolution, but otherwise identical parameters) Hc1b15b, representing windtunnel and free-fall models, and demonstrating resolution effects as well as longterm evolution. Figure 3 summarizes the time history of the three models, from top to bottom the mass, velocity, Mach number, ratio of thermal over dynamical time scales and the number of grid cells against time. We will discuss each of them in turn. To measure the time histories of the clouds, we ran a core finder identifying coherent structures in three dimensions with temperatures $T<10^{4} \mathrm{~K}$ as a proxy for neutral hydrogen. Structures with fewer than 64 cells are not counted. In the following, we will use "cloud mass" synonymously with "cloud mass in HI".

\subsubsection{Mass evolution}

We begin our discussion with the mass histories (top panels of Fig. 3), which indicate the survival chances of the model cloud. The simulations start out with one cold cloud, which subsequently fragments due to a combination of dynamical and thermal instabilities. The thick solid line denotes the total mass in HI, while the thin solid line follows the mass evolution of the most massive fragment (usually the main cloud except for very late stages of nearly complete fragmentation). The dashed line indicates $10 \%$ of the initial cloud mass. The centerof-mass velocity of each fragment is shown in color.

With increasing time, more and more material is ablated from the cloud, resulting in a growing number of cloud fragments. The increasing lower mass envelope of the fragments in model Hc1b15b (to some extent visible in Hc1b13a as well) results from the recooling of the fragments. With decreasing $z$, the cloud fragments are being compressed by the increasing background pressure, thus leading to higher cooling rates and "re-formation" of cold HI-cloudlets. The mass fraction in the small, "re-formed" cloudlets amounts to $\approx 10 \%$ of the HI-mass of the original cloud. This effect is absent in the wind-tunnel experiments, where the cloud is being shredded into successively smaller fragments. The evolution of "real" HVCs we expect to lie in between these two extremes of exponential density increase and constant density of the back- 

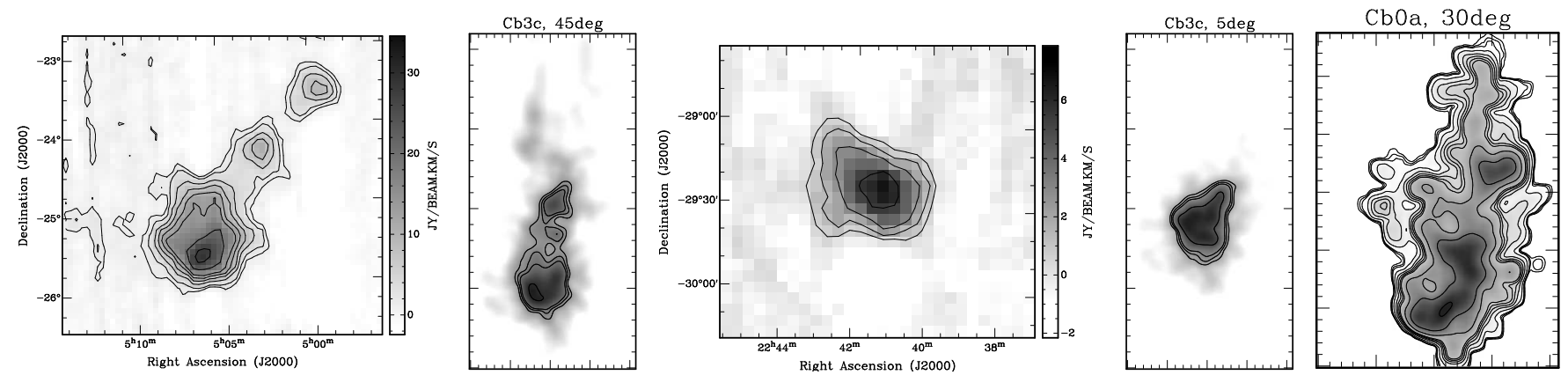

FIG. 2.- Column density maps of observed and modeled HVCs. Observed clouds were taken from the HIPASS catalogue by Putman et al. (2002). From left to right: HIPASS cloud \#648, model cloud Wb1a15b at 45 Myr, rotated by $45^{\circ}$, HIPASS cloud \#200, model cloud Wb1a15b at $45 \mathrm{Myr}$, rotated by $5^{\circ}$, and model cloud We1c05b at $110 \mathrm{Myr}$, rotated by $30^{\circ}$. Contours indicate column densities levels identical for all clouds, at $(1.2,1.6,2,4,8,12,16,20) \times 10^{18} \mathrm{~cm}^{-2}$. Because of the higher peak densities, model We1c05b has additional contour levels at $(40,80,160) \times 10^{18} \mathrm{~cm}^{-2}$. The models have a nominal resolution of $\approx 2 \mathrm{pc}$, corresponding to $0.7^{\prime}$ at $10 \mathrm{kpc}$. The maps have been degraded by a Gaussian corresponding to an angular resolution of $3.0^{\prime}$ for a more realistic comparison to observations.
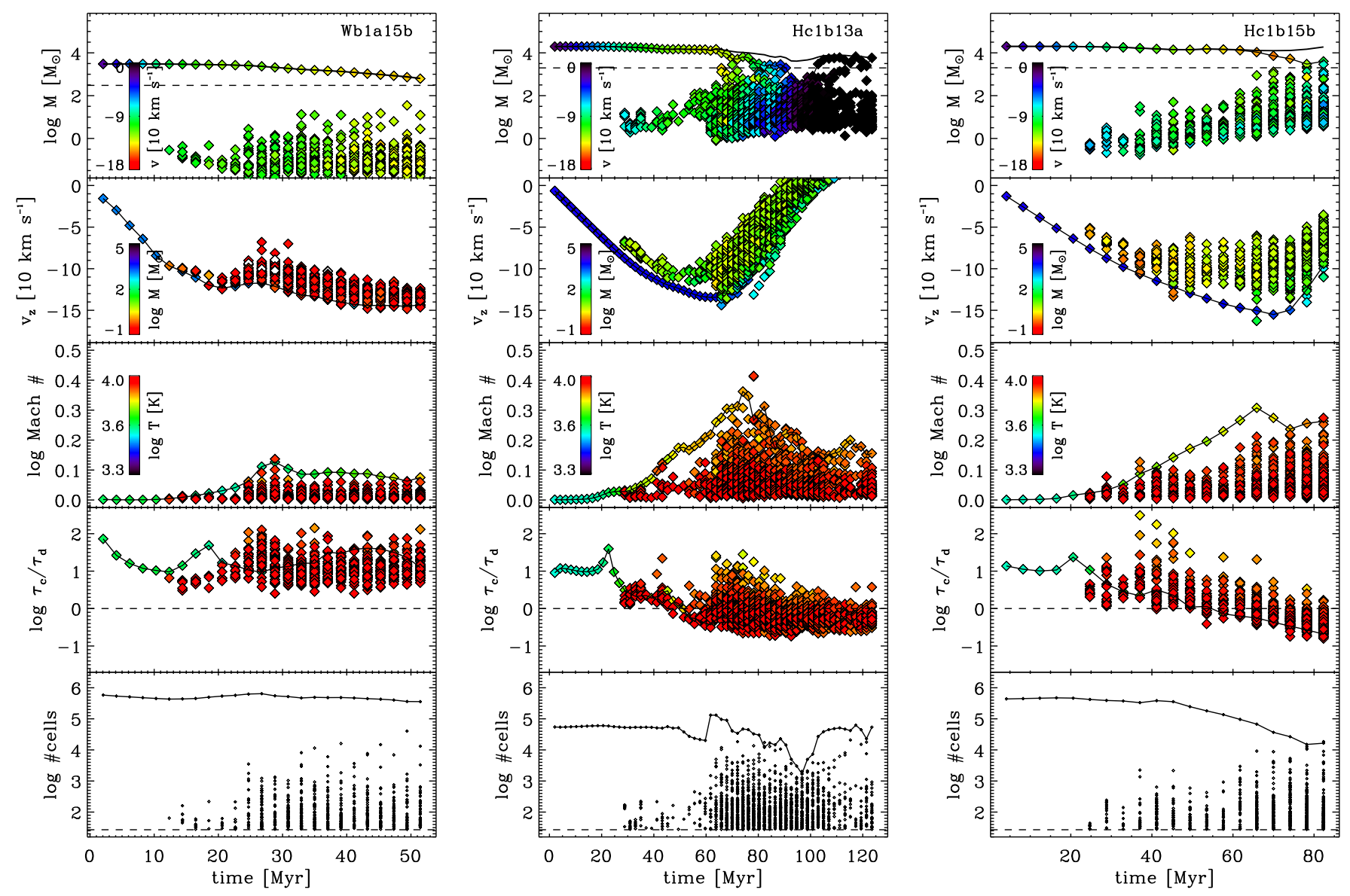

FIG. 3.- Time histories for models Wb1a15b, Hc1b13a and (at twice the resolution) Hc1b15b. From top to bottom: logarithm of HI mass of all cloud fragments, their velocity, the internal Mach number of each cloud fragment, the ratio of cooling time scale over dynamical timescale to estimate the likelihood for disruption, and the number of cells in each fragment. Color codes as indicated in the panels. Note that the velocities are given in $10 \mathrm{~km} \mathrm{~s}^{-1}$. The ratio $\tau_{c} / \tau_{d}$ is color-coded with the temperature as in the Mach number plot. Solid lines denote the evolution of the most massive fragment (i.e. the main body of the cloud). For the mass-history, a thick solid line shows the total HI mass. The dashed line at the bottom of the cell number plot indicates the threshold of 64 particles required to be accepted as a "cloud". Note that this is far below the resolution limit for hydrodynamical instabilities (see discussion in 93.4). 
Heitsch \& Putman

TABLE 1

Model Parameters

\begin{tabular}{ccccccccccc}
\hline \hline Name & $N_{x}$ & $\begin{array}{c}n_{h} \\
\mathrm{~cm}^{-3}\end{array}$ & $\begin{array}{c}n_{c} \\
\mathrm{~cm}^{-3}\end{array}$ & $\begin{array}{c}v_{0} \\
\mathrm{~km} \mathrm{~s}^{-1}\end{array}$ & $\begin{array}{c}M_{0} \\
10^{3} \mathrm{M}_{\odot}\end{array}$ & $\begin{array}{c}N_{0} \\
10^{19} \mathrm{~cm}^{-2}\end{array}$ & $\begin{array}{c}R_{0} \\
\mathrm{pc}\end{array}$ & $\begin{array}{c}R n \\
\mathrm{cells}\end{array}$ & $\begin{array}{c}\tau_{0} \\
\mathrm{Myr}\end{array}$ & $\begin{array}{c}D_{0} \\
\mathrm{kpc}\end{array}$ \\
\hline Wa1b10b & 256 & $1 \times 10^{-4}$ & 0.1 & 100 & 1.4 & 1.32 & 21 & 36 & 35 & 3.3 \\
Wa3b10b & 256 & $1 \times 10^{-4}$ & 0.3 & 100 & 4.5 & 3.96 & 21 & 36 & 65 & 5.9 \\
Wb1a15a & 128 & $3 \times 10^{-5}$ & 0.1 & 150 & 5.9 & 1.32 & 50 & 32 & 50 & 5.4 \\
Wb1a15b & 256 & $3 \times 10^{-5}$ & 0.1 & 150 & 3.0 & 1.32 & 50 & 51 & 59 & 7.2 \\
We1c05a & 128 & $3 \times 10^{-4}$ & 0.1 & 50 & 44 & 2.94 & 150 & 38 & 150 & 5.3 \\
We1c05b & 256 & $3 \times 10^{-4}$ & 0.1 & 50 & 44 & 2.94 & 150 & 77 & 162 & 6.0 \\
We1c09a & 128 & $3 \times 10^{-4}$ & 0.1 & 90 & 44 & 2.94 & 150 & 38 & 81 & 5.4 \\
We1b10a & 128 & $1 \times 10^{-4}$ & 0.1 & 100 & 44 & 2.94 & 150 & 38 & 64 & 3.2 \\
Hc1b13a & $128^{a}$ & $1 \times 10^{-4}$ & 0.1 & 130 & 20 & 2.55 & 89 & 46 & 92 & 9.0 \\
Hc1b15b & 256 & $1 \times 10^{-4}$ & 0.1 & 150 & 20 & 2.55 & 89 & 92 & 68 & 9.2 \\
Hc2b15a & $128^{a}$ & $1 \times 10^{-4}$ & 0.2 & 150 & 40 & 5.10 & 89 & 46 & 39 & 6.7 \\
Ha1b06a & 128 & $1 \times 10^{-4}$ & 0.2 & 60 & 7.3 & 1.32 & 21 & 18 & 36 & 1.8 \\
Hd1b17a & $128^{a}$ & $1 \times 10^{-4}$ & 0.1 & 170 & 37 & 2.87 & 133 & 34 & 103 & 11 \\
Hd1b16b & $256^{a}$ & $1 \times 10^{-4}$ & 0.1 & 160 & 37 & 2.87 & 133 & 68 & 74 & 11 \\
Hd1b21a & $128^{a}$ & $1 \times 10^{-4}$ & 0.1 & 210 & 37 & 2.87 & 133 & 34 & 103 & 12 \\
\hline
\end{tabular}

Note. - 1st column: model name (see text), 2nd: number of grid cells along shorter domain axes $(a=128, b=256$ cells $)$, 3rd: background halo density $\left(a=3 \times 10^{-5}, b=1 \times 10^{-4}\right.$, $\left.c=3 \times 10^{-4} \mathrm{~cm}^{-3}\right)$, 4th: cloud peak density, 5th: nominal cloud velocity, 6 th: initial cloud mass, 7th: initial peak column density, 8th: initial cloud radius $(a=21, b=50, c=89, d=133$, $e=150 \mathrm{pc}$ ), 9th: resolution elements per cloud radius, 10th: characteristic life time (eq. 3), 11th: characteristic distance traveled (eq. 4).

$a$ These models have been regridded (see $\frac{12.2 .1}{4}$ ) 
ground medium, since the cloud trajectories will generally not be restricted to motions along $z$, specifically if the clouds stem from material stripped off satellites.

\subsubsection{Peak Velocities}

The second row of panels in Figure 3 complements the mass histories discussed above. Although this information may seem redundant, it is easier to glean the peak velocities reached, and the velocity distribution of the fragments. As in the mass histories, the thin solid line traces the evolution of the most massive fragment.

The velocities of the cloud fragments are generally smaller than that of the main body, indicating a growing "tail" of the cloud, due to dynamical drag. The lag is consistent with the velocity gradients in observed HVCs (Brüns et al. 2000, 2001; see also Westmeier et al.|2005).

While for the wind-tunnel experiment, the velocity is prescribed, the velocities reached by the $\mathrm{H}$-series range well below ballistic velocities (see e.g. Beniamin \& Danly 1997), and rarely exceed $\approx 150 \mathrm{~km} \mathrm{~s}^{-1}$. Several effects play a role here. First, because the clouds are deformed due to hydrodynamical interaction with the background gas, the drag forces might be higher than expected. Second, the clouds lose mass and eventually disintegrate, and third, the increasing background density leads to stronger drag. In fact, for model Hc1b13a, the combination of mass loss and background density increase brings the cloud fragments to a halt at around $100 \mathrm{Myr}$. Together with the recooling of cloudlets (see previous section), this offers an interesting possibility of reforming HI-clouds close to the disk (4.3).

Observationally, relative to the Galactic standard of rest (GSR), the maximum $\mathrm{HVC}$ velocities are $\pm 250 \mathrm{~km} \mathrm{~s}^{-1}$, with the more typical GSR velocities $<100 \mathrm{~km} \mathrm{~s}^{-1}$ in magnitude and a median value of $-30 \mathrm{~km} \mathrm{~s}^{-1}$ (e.g. Wakker \& van Woerden 1991; Putman et al. 2002). Though we do not know the true velocities of the clouds, this represents our only observational constraint. Many people have begun to use the deviation velocity, or how much the HVC motions differ from a simple model of Galactic rotation, when discussing HVCs (e.g. Wakker 2004). The majority of the deviation velocities are $<200 \mathrm{~km} \mathrm{~s}^{-1}$. The peak values of $\pm 300 \mathrm{~km} \mathrm{~s}^{-1}$ belong to HVCs associated with the Magellanic System.

\subsubsection{Internal Mach Numbers}

The third row of Figure 3 shows the logarithm of the internal Mach number for all identified fragments. We define the Mach number as the density-weighted rms velocity within a fragment over its average sound speed. As an estimate of the sound speed, we show the volume-averaged temperature of each fragment in color. Note that the lowest temperatures can reach $100 \mathrm{~K}$ in our models, in agreement with observed values (Schwarz \& Wakker 2004).

The Mach numbers reach values up to 2, although it should be noted that these are averaged over the cloudlets - in the coldest regions, the Mach numbers can exceed values of 5 . Thus, the clouds have strong internal dynamics, indicating dynamical instabilities at work. Yet the Mach numbers and disruption timescales do not correlate - for that our measures at this point are too crude, and we defer a more detailed discussion to a future paper.

\subsubsection{Thermal over Dynamical Timescales}

The ratio of the cooling time over the dynamical timescale $\tau_{c} / \tau_{d}$ can be used as a measure for the importance of thermal over dynamical instabilities. Cooling can stabilize the cloud against disruption by damping the propagation of waves into the cloud (e.g. Vietri et al. 1997). The cooling timescale is given by $\tau_{c} \equiv k_{B} T /(n \Lambda)$, and as the dynamical timescale we take the sound crossing time $D / c_{s}$, where the diameter $D$ is estimated by taking the geometric mean of a cloud fragment's extent along the three grid axes, i.e. $D \equiv\left(D_{x} D_{y} D_{z}\right)^{1 / 3}$. For $\tau_{c} / \tau_{d}>1$, the cooling time is longer than the sound crossing time, and perturbations will only be slightly damped when traveling into the cloud. For $\tau_{c} / \tau_{d}<1$, thermal effects dominate, leading to a stabilization of the cloud. If the densities and temperatures of the cloud fragments were constant with time, dynamical effects should eventually win over thermal ones with progressive fragmentation of the cloud.

Cloud Wb1a15b is completely dominated by dynamics $\left(\tau_{c} / \tau_{d}>1\right.$ for all $\left.t\right)$. This is consistent with the gradual disruption of the cloud mirrored by the continuous mass loss (top panel). As the number of cells in the most massive fragment (bottom panel) stays nearly constant, the mean cloud density decreases, thus further lowering the importance of thermal effects.

Clouds Hc1b13a and Hc1b15b are initially dominated by dynamics, however, the increasing background pressure compresses the cloud and thus shortens the cooling time, rendering some of the fragments and eventually the main body of the cloud thermally dominated for $t \gtrsim 45$ Myr. Yet the cloud still disrupts because the dense gas enters the thermally unstable regime of the cooling curve. Fragmentation is enhanced since the condensation mode of the thermal instability has an outer length scale set by the product of the sound crossing time and the cooling time (Burkert \& Lin 2000; Heitsch \& Hartmann 2008).

\subsubsection{Cloud Resolution}

The bottom panels of Figure 3 show the number of cells in each cloud (fragment). The dashed line denotes a limit of 64 cells below which a fragment is not counted as a cloudlet any more. Clearly, this number is well below the hydrodynamical resolution limit (see discussion in (3.4). For the vast majority of the time, the main body of the clouds stays above $10^{4}$ cells. The jump at $60 \mathrm{Myr}$ in model Hc1b13a is due to the regridding of the model (see 2.2.1).

\subsubsection{Cloud Travel Distances}

Figure 4 shows the mass and velocity history in terms of the cloud's position $z$ above the Galactic plane, for models Wb1a15b, Hc1b13a and Hc1b15b. Strictly, for model Wb1a15b, the initial position is arbitrary. Also note that the cloud velocities for the $\mathrm{W}$-series are not self-consistent, but are imposed by the wind entering the simulation domain (see 2.2.1). For the H-series, the velocity profiles of the most massive fragment (i.e. the main body of the cloud) are initially similar to those derived 
by Benjamin \& Danly (1997) (their Fig. 3) for clouds with a column density of $10^{19} \mathrm{~cm}^{-2}$ being dropped at $10 \mathrm{kpc}$. Yet our clouds do not reach terminal velocities, but are successively decelerated due to continuous mass loss. As demonstrated by models Hc1b13a and Hc1b15b, the more massive fragments are to be found at lower $z$, and - as discussed above - the fragments lag behind the main body of the cloud. This should be observable as a gradient along head-tail clouds or a shift in velocity components between warm and cold gas (Brüns et al. 2000, 2001).

\subsection{Characteristic Disruption Times and Distances}

Our model data allow us to estimate characteristic timescales $\tau_{0}$ and distances $D_{0}$ within which a cloud of a given mass and at a given background halo density will lose its HI content. This information we then can use to predict whether observed HVCs with distance constraints will retain any of their HI, or whether they will disintegrate before they reach the disk. The time series of our models provide us with an opportunity to sample a whole range in cloud mass and (for series $\mathrm{H}$ ) in halo densities.

The disruption time and distance are given by

$$
\tau_{0} \equiv \frac{M}{d M / d t}
$$

and

$$
D_{0} \equiv \frac{M}{d M / d z},
$$

with the cloud mass $M$, the mass loss rate with respect to time $d M / d t$ and distance $d M / d z$. The mass range is sampled by taking the differences (here for time)

$$
\frac{d M}{d t} \equiv \frac{M(t)-M_{e n d}}{t-t_{e n d}}
$$

for all model times $t>t_{\text {end }}$, where $t_{\text {end }}$ is the smaller of the time at which the cloud has lost $90 \%$ of its original HI content, and the end of the simulation. Figure 5] summarizes $D_{0}$ and $\tau_{0}$ in dependence of the cloud mass, for all models. Colors indicate the velocity $v(t)$ of the cloud.

Most clouds are disrupted within $10 \mathrm{kpc}$ and $100 \mathrm{Myr}$, with the majority surviving for not longer than $8 \mathrm{kpc}$. Slower clouds survive for longer times and distances, mirroring the lower mass-loss rates, with clouds at $v_{z} \gtrsim$ $100 \mathrm{~km} \mathrm{~s}^{-1}$ lasting for at most $8 \mathrm{kpc}$. Higher background halo densities also lead to faster disruption, as expected.

Figure 6 can be used to predict the disruption distances $D_{0}$ for observed HVCs, given their mass, the halo background density $n_{h}$ and the cloud velocity $v$. The latter two have been combined into the ram pressure (in $\mathrm{K} \mathrm{cm}^{-3}$ ). The left panel of the Figure shows the disruption distance $D_{0}$ at a given mass and (in grayscale) ram pressure of all models, derived from Figure 5. Overplotted are the masses and distances above the plane $z$ for a sample of HVCs taken from the extended catalogue by Wakker \& van Woerden (1991) with $\sim 30^{\prime}$ resolution, and from a catalog of the clouds at the tail of Complex $\mathrm{C}$ as observed with Arecibo by $\mathrm{Hsu}$ et al. (2009; $3.5^{\prime}$ resolution). We selected only clouds of HVC complexes with known distance constraints (see Tab 2). Masses have been calculated from the distance constraints and the
TABLE 2

Distance Constraints

\begin{tabular}{lccc}
\hline \hline \multicolumn{1}{c}{ Complex } & $D[\mathrm{kpc}]$ & $z[\mathrm{kpc}]$ & reference \\
\hline ACHV/Cohen Strm & $5.0-11.7$ & $0.9-9.4$ & $(1)$ \\
GCP/Smith Cld & $9.8-15.1$ & $2.6-10.1$ & $(1)$ \\
$\mathrm{A}$ & $4-10$ & $2.2-7.7$ & $(2)$ \\
$\mathrm{M}$ & $1.5-4.4$ & $1.1-4.0$ & $(3)$ \\
$\mathrm{C}$ & $10.0 \pm 2.5$ & $0.9-11.0$ & $(4,5)$ \\
WB & $8.8_{-1.3}^{+2.3}$ & $1.7-10.2$ & $(6)$ \\
\hline
\end{tabular}

Note. - 1st column: complex name, 2nd column: heliocentric distance, 3rd column: distance towards Galactic plane, 4th column: reference: (1) Wakker et al. (2008), (2) van Woerden et al. (1999), (3) Danly et al. (1993), (4) Thom et al. (2008), (5) Wakker et al. (2007), (6) Thom et al. (2006). The ranges in $z$ have been estimated using fiducial distances $D$ of $8.4,12.5,7.0$, and $3.0 \mathrm{kpc}$ for complexes with $D$-ranges given, and then accounting for the full extent in Galactic latitude.

total flux per cloud as $M_{H I}\left[\mathrm{M}_{\odot}\right]=2.36 \times 10^{-1} D_{k p c}^{2} F$ $\left[\mathrm{Jy} \mathrm{km} \mathrm{s}^{-1}\right]$.

Clearly, model clouds with higher mass tend to travel farther distances until their HI content is completely gone - indicated by the upper envelope of the grayscale map. This effect is offset by an increased ram pressure - an increase by a factor of 10 reduces the disruption length scale $D_{0}$ by approximately the same factor.

Figure 6 shows that many of the observed HVCs will not make it to the disk in cold form. The left panel is restricted to the mass range sampled by our model HVCs. Clouds located above the gray shaded region will clearly disrupt before making it to the disk, while clouds within the Gray shaded region may make it depending on the exact halo density they are moving through and their velocity. Yet since more than $90 \%$ of the mass in the HVC complexes with distance constraints resides in clouds of large masses (as catalogued by Wakker \& van Woerden 1991), the right panel of Figure 6 shows an extrapolation of the ram pressure map to larger masses. The extrapolation has been calculated by a two-dimensional quadratic fit to the ram pressure surface shown in the left panel. Although this approach is rather crude, it demonstrates the salient correlation between $D_{0}, M$ and $P_{\text {ram }}$. While the smaller HVCs will most likely be disrupted on their way to the disk, the situation is less clear for the main body of the larger complexes at these distances. Yet it should be noted that when higher resolution catalogs are used, these massive clouds are often broken up into smaller clouds (Putman et al. 2002; Fig. 1 of Schwarz \& Wakker 2004). In other words, the fact that the substructure in $\mathrm{HVC}$ complexes tends to be underestimated renders the predicted disruption distances of Figure 6 as upper limits.

Figure [7 offers a straight-forward way ${ }^{3}$ to read off the disruption length scale $D_{0}$ as a function of $\log M$ and the ram pressure $\log P$. This has been derived by inverting

\footnotetext{
3 We thank the referee for the suggestion.
} 

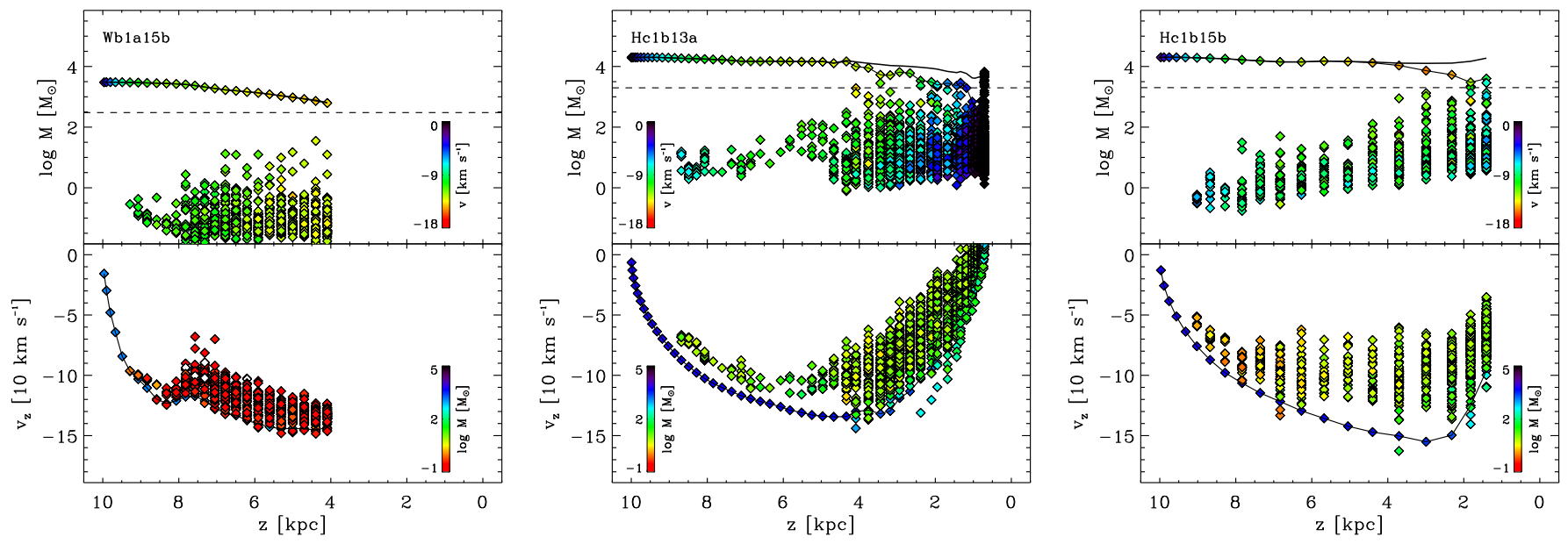

FIG. 4.- Mass (top) and velocity (bottom) of cloud fragments against distance from Galactic plane for models Wb1a15b, Hc1b13a and Hc1b15b. Note that the velocities are given in $10 \mathrm{~km} \mathrm{~s}^{-1}$. Solid lines denote the evolution of the most massive fragment (i.e. the main body of the cloud). For the mass-history, a thick solid line shows the total HI-mass. Because of the small mass contained in the fragments of model Wb1a15b, the lines are indistinguishable for that model.

the two-dimensional quadratic fit, yielding

$$
\begin{gathered}
D_{0}=3.481 \log M-(-165.2+77.16 \log M \\
\left.-18.54(\log M)^{2}+86.89 \log P\right)^{1 / 2} \\
{[\mathrm{kpc}],}
\end{gathered}
$$

where $M$ is given in $M_{\odot}$, and $P$ in $\mathrm{K} \mathrm{cm}^{-3}$. Since the quadratic form represents the data reasonably well only in a limited range of parameters, equation (6) is limited to the range in $M$ and $P$ indicated by the outer contours in Figure 7 . Within that range, equation (6) is accurate to approximately $10 \%$. Below the thick line towards high masses and low pressures (for $D_{0}$ exceeding $16 \mathrm{kpc}$ ), equation (6) breaks down. Still, it allows us to read off the approximate disruption length scale $D_{0}$ for physically reasonable values of $M$ and $P$.

\subsection{Resolution issues}

Numerical resolution is a main concern when modeling the disruption of clouds by dynamical instabilities. In their two-dimensional study of cloud disruption by passing shocks, Klein et al. (1994) quote a resolution of approximately 100 cells per cloud radius (or $R 100)$ to fully resolve the flow dynamics around the cloud. Yet Korycansky et al. (2000) noted that since the dynamical instabilities lead to non-linearities and turbulence, detailed convergence might be unfeasible (see also Mac Low \& Zahnle 1994). They argue for statistical convergence (or convergence of integrated quantities) at $\approx R 100$. In a similar vein, Roulston \& Ahrens (1997) argued in the context of the disruption of comets in planetary atmospheres that $R 25$ is sufficient to converge on the penetration depth within a scale height.

Checking the lower panels of Figure 3, it is obvious that our models will have a hard time meeting the resolution criterion of $R 100$ for all of the cloud fragments, especially at later times when the main body of the cloud will develop a strong tail, reducing its cross section and thus possibly suppressing Rayleigh-Taylor instabili- ties (Roulston \& Ahrens 1997; Korycansky et al. 2000). Clearly, the cloud fragments will be dynamically underresolved, an effect which is exacerbated in the models of the H-series, due to the increasing external pressure. The smallest cloudlets might be dominated by evaporation (e.g. Stanimirovic et al. 2008 in the context of the Magellanic Stream).

Figure 8 helps us to assess the effect of $R n$ on the mass loss rate in terms of time and distance traveled (eqs. 314). The mass loss rates will be correlated with the strength of the dynamical instabilities disrupting the cloud (e.g. Nulsen 1982; Korvcansky et al. 2000), which in turn will be suppressed in underresolved models. No correlation between mass loss rates and resolution is discernible, if we ignore the clearly underresolved model Ha1b06a. Although this clearly does not mean that the models are fully resolved, we are confident that they are sufficiently resolved for our purposes.

\subsection{Differences to Previous Numerical Work}

The wind-tunnel experiments (series W) are conceptually similar to the models discussed by Quilis \& Moore (2001). Consistent with their claim, we do not notice a substantial difference in disruption timescales between models with and those without cooling ${ }^{4}$. However, for series $\mathrm{H}$, the comparison models without cooling showed substantially shorter disruption times and distances, since the cloud suffers adiabatic compression and heating due to the increasing background pressure. Thus, the initially cold material is rapidly heated, and thus "lost" (i.e. not identified as HI any more). In that sense, cooling is relevant for the longer survival of the clouds of series $\mathrm{H}$, and it is obviously indispensable for the recooling observed in e.g. model Hc1b13a.

Santillan et al. (2004) studied the impact of HVCs on the Galactic disk using three-dimensional isothermal magneto-hydrodynamical (MHD) models. Focusing on the physics of the impact, they had the HVC start at

4 Although we ran a few comparison models without cooling, we decided not to discuss them in more detail, since their restricted physics render them irrelevant for our purposes. 


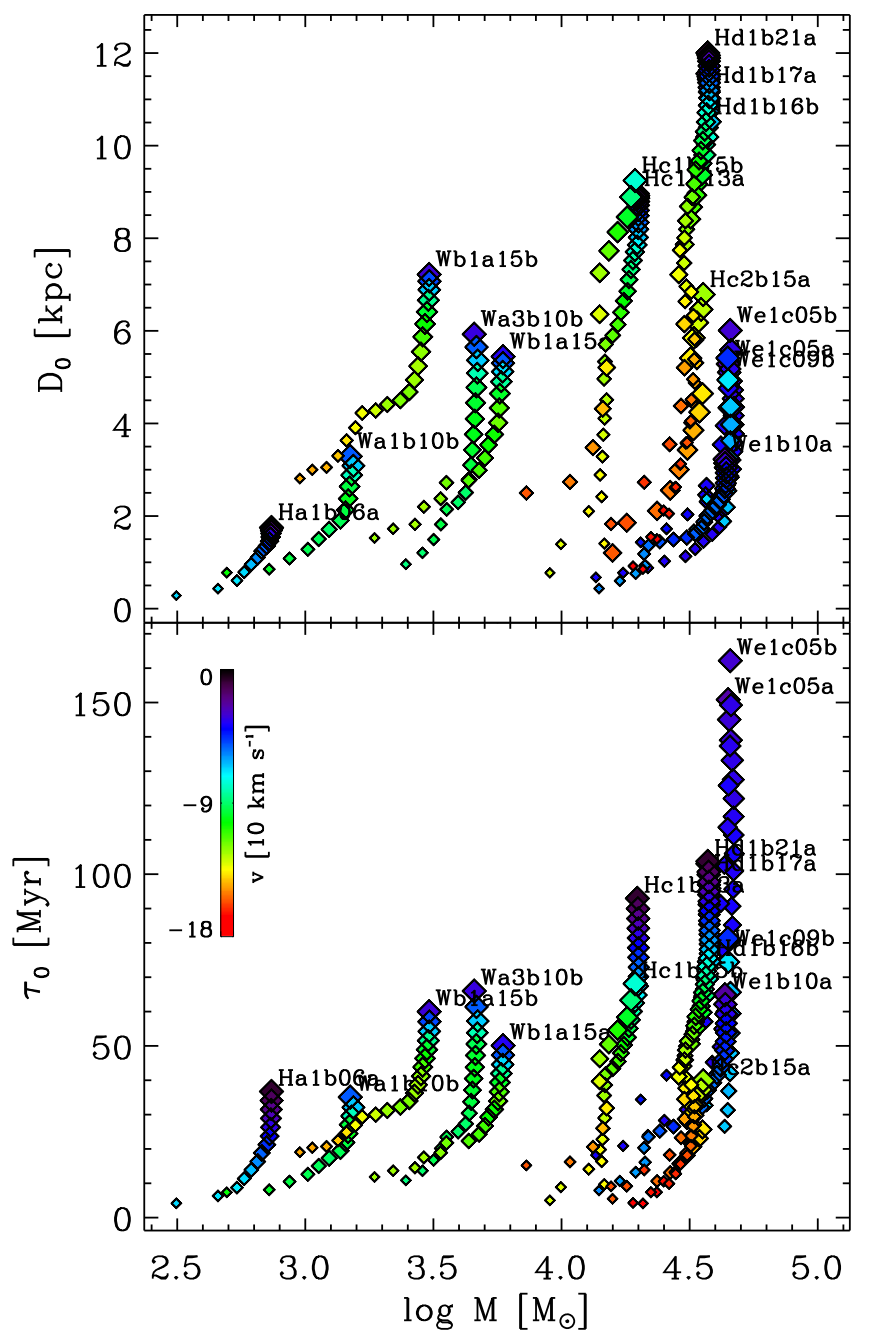

FIG. 5.- Distance $D_{0}$ and timescale $\tau_{0}$ within which a cloud of given mass will lose its total HI content. Each "track" represents the time sequence of one model, with each point indicating $D_{0}$ or $\tau_{0}$ of the largest fragment (see eqs. 34 and text). Thus, the disruption rate $d M / d t$ increases over time. Colors denote the cloud velocities, showing that faster clouds are shorter-lived. Symbol sizes indicate model times, with large symbols for early times, and small ones for late times. Note that $D_{0}$ is not a distance above the plane, but a disruption length scale.

$z=2.5 \mathrm{kpc}$ above the plane. Thus, conclusions on the possibly stabilizing effects of magnetic fields on HVCs over long distances could not be addressed.

A related problem albeit in a different physical regime was considered by Ruszkowski et al. (2007) who studied the disruption of buoyant bubbles driven by AGN feedback in galaxy clusters. They found that tangled magnetic fields can prevent the disruption of the bubbles if the coherence length of the field is larger than the bubble size, i.e. if the field is only weakly tangled with respect to the bubble scale. Otherwise the bubbles are quickly shredded.

Bland-Hawthorn et al. (2007) focus on the origin of the $\mathrm{H} \alpha$ emission along the Magellanic Stream and explain it by a model of successively shocked material as it is ablated from clouds in the Stream predominantly by Kelvin-Helmholtz instabilities (see however Murali 2000). Since they are interested in the dynamics of the
Stream's gas, they start with a two-phase medium, with the cold phase having a fractal distribution. Their numerical method includes non-equilibrium cooling. They speculate that much of the gas accretion necessary to fuel Galactic star formation happens in the warm ionized phase, largely of material shorn off the Magellanic Stream (see also Bland-Hawthorn 2008). Clearly, as shown above, HI clouds originating at the distance of the Magellanic Stream will not survive the trip to the disk.

\section{DISCUSSION}

\subsection{Interpretation of Morphologies}

The model clouds usually develop a pronounced tail, visible not only in the maps (Fig. 2), but also in the velocity lags of the smaller HI fragments (Figs. 314). The lag is consistent with the velocity gradients and/or shifts between warm and cold gas components in observed HVCs (Brüns et al. 2000, 2001; see also Westmeier et al. 2005) and should be examined further in higher resolution surveys with Parkes and Arecibo. The head-tail structure will be most pronounced when the trajectory of the cloud is perpendicular to the line-of-sight. HVCs that appear round may show a kinematic offset in their cold and warm components providing additional evidence of clouds being destroyed by the surrounding halo medium. In the future it should be possible to create a grid of the typical length/velocity offset expected for clouds at different stages of destruction and viewing angles.

\subsection{Interpretation of Timescales and Distances}

From Figure 5 we see that the majority of our model clouds will be disrupted within $\lesssim 8-10 \mathrm{kpc}$. However, various physical and numerical issues will affect our estimates.

\subsubsection{Effects Reducing $\tau_{0}$ and $D_{0}$}

The following effects will render our estimates as upper limits.

(1) - The actual halo density may be larger than our maximum of $n_{h}=3 \times 10^{-4} \mathrm{~cm}^{-3}$. Gaensler et al. (2008) set limits on the halo density at $z>5 \mathrm{kpc}$ using the 6 measurements they have that fit that criterion. They find a $3 \sigma$ upper limit of $n_{h}<7.6 \times$ $10^{-4} \mathrm{~cm}^{-3}$. This type of limit is consistent with other work based on pulsar dispersion measures (e.g. Howk et al. 2006). The majority of the constraints on the halo density are integrated with assumptions on the temperature and structure of the halo. To explain observations of OVI absorption, Sembach et al. (2003) propose densities $\lesssim 10^{-4}$ down to $10^{-5} \mathrm{~cm}^{-3}$. Peek et al. (2007) find consistency with a density of a few $10^{-4} \mathrm{~cm}^{-3}$ at about $10 \mathrm{kpc}$, and Grcevich \& Putman (2009) estimate that a halo density $>10^{-4} \mathrm{~cm}^{-3}$ is likely at these types of distances to explain dwarf spheroidal stripping. Bregman \& Lloyd-Davies (2007) and Sanders et al. (2002) combine X-ray absorption and emission measures and find a density of $9 \times 10^{-4} \mathrm{~cm}^{-3}$ for a lengthscale of $20 \mathrm{kpc}$. See also Bregman (2007) for a discussion.

(2) - The inflow gas density in the $\mathrm{W}$-series is constant, i.e. we do not take into account the increase of the gas 

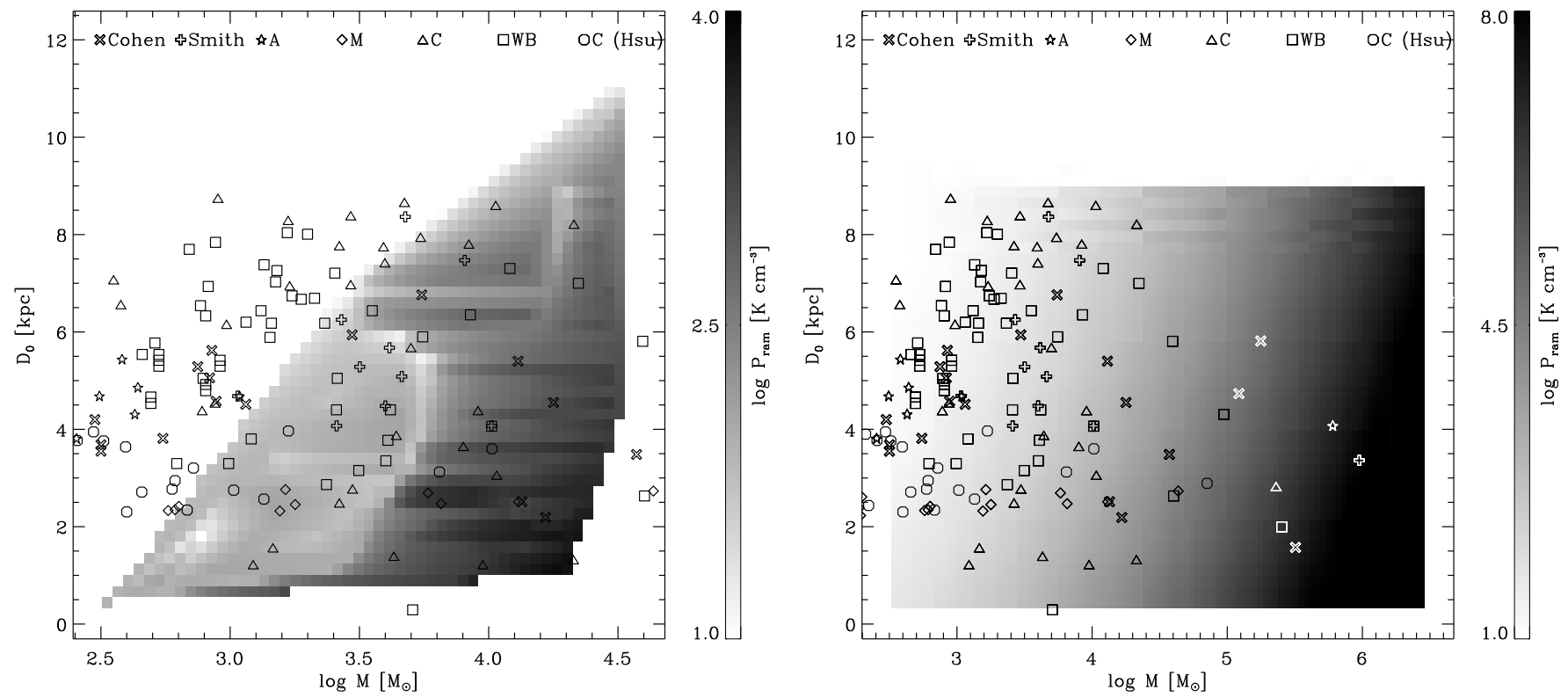

Fig. 6. - Left: Disruption distance $D_{0}$ derived from models shown in Fig. [5 against cloud mass $M$, for a range of background halo densities $n_{h}$ and cloud velocities, combined into a ram pressure $m_{H} n_{h} v^{2} / k_{B}$ (see colorbar). A ram pressure of $10^{4} \mathrm{~K} \mathrm{~cm}^{-3}$ corresponds to approximately $100 \mathrm{~km} \mathrm{~s}^{-1}$ at a background halo density of $10^{-2} \mathrm{~cm}^{-3}$. Symbols represent the $z$ height above the plane and mass of clouds that are part of HVC complexes with known distance constraints (see Tab. 2). Right: Similar to left panel, but using a two-dimensional quadratic fit to the ram pressure surface, and extrapolating to higher masses to estimate the disruption distances for more massive clouds. Except for the main body of Complex $\mathrm{C}\left(\right.$ at $\left.\approx 3 \times 10^{6} \mathrm{M}_{\odot}\right)$, all clouds of the observational comparison sample are shown.

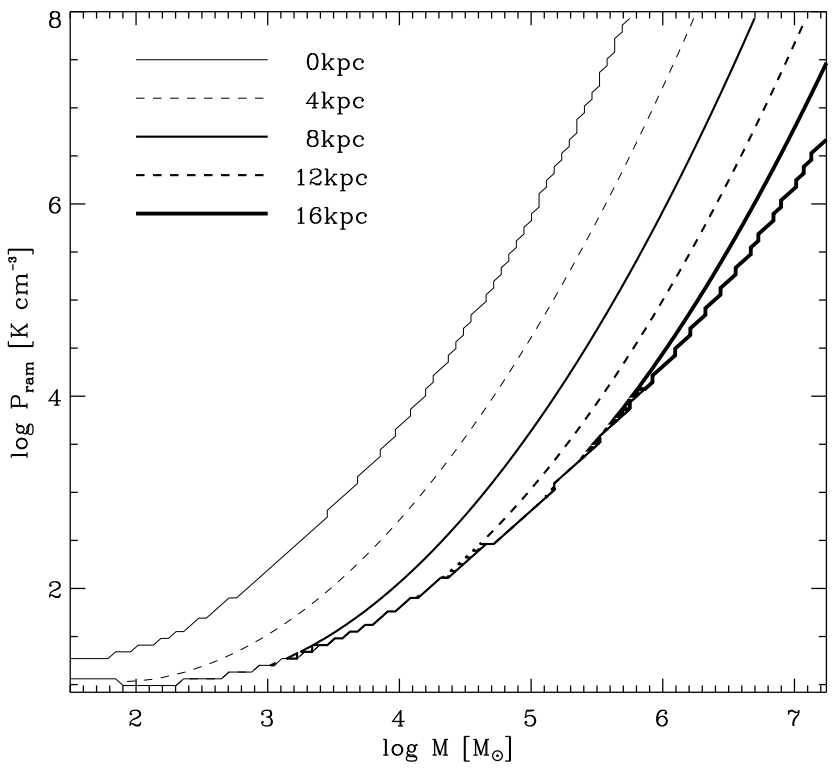

FIG. 7.- Contours of the disruption length scale $D_{0}$ in kpc, as a function of the cloud mass $M$ and the ram pressure $P$. A ram pressure of $10^{4} \mathrm{~K} \mathrm{~cm}^{-3}$ corresponds to approximately $100 \mathrm{~km} \mathrm{~s}^{-1}$ at a background halo density of $10^{-2} \mathrm{~cm}^{-3}$. The range of applicability of equation (6) is indicated by the 0-line towards higher $P$ and lower $M$, and the thick line towards lower $P$ and higher $M$ (i.e. $D_{0} \lesssim 16 \mathrm{kpc}$ ).

density along the trajectory of the cloud towards smaller $z$ for those models.

(3) - The velocity of our clouds gradually increases to the velocity listed in Table 1, so any initial velocity from the origin of the cloud - e.g. the motion of the Milky Way satellite - is not included.

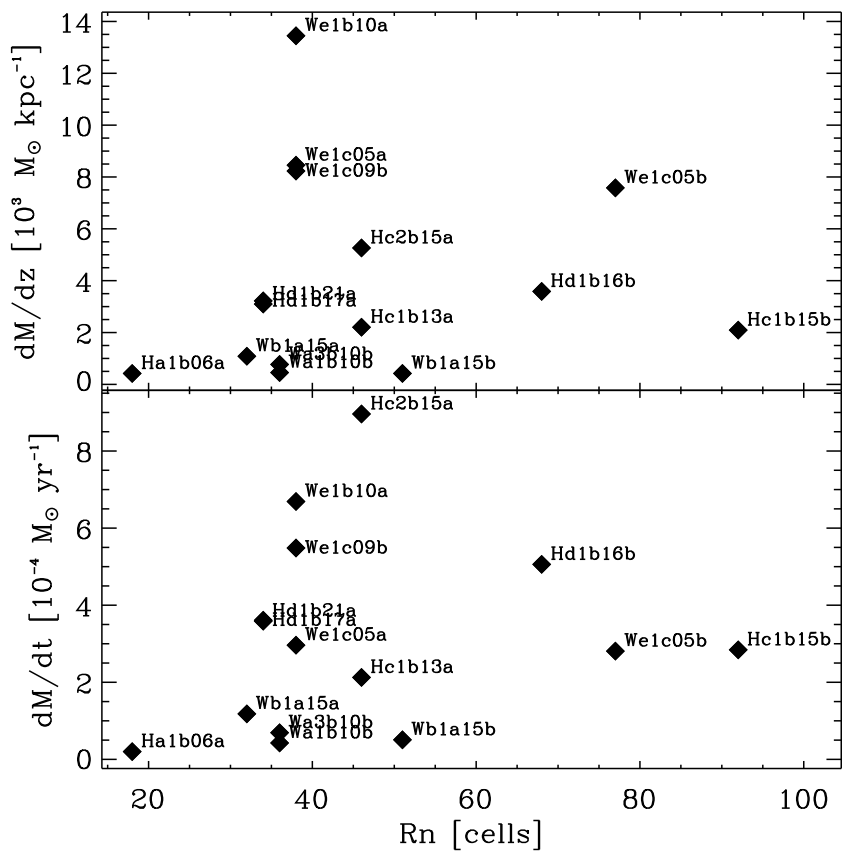

Fig. 8.- Mass loss rate in terms of time (bottom) and distance traveled (top), against the number of resolution elements per radius $R n$. Ignoring the clearly underresolved model Halb06a, there is no trend of mass loss rates with resolution.

(4) - Resolution effects - in the H-series exacerbated by the increased cooling due to the rising external pressuretend to work in favor of faster disruption: under-resolved clouds tend to take longer to disrupt (Roulston \& Ahrens 1997; Korvcansky et al. 2000; see also Fig. 8).

(5) - Assuming that the cooling timescale does not substantially decrease during the lifetime of the cloud, break- 
ing the cloud into successively smaller fragments should render $\tau_{c} / \tau_{d}>1$ eventually, i.e. the fragments should be more likely to disrupt due to dynamical instabilities, and eventually through evaporation (Cowie \& McKee 1977).

(6) - The substructure in HVCs and complexes tends to be underestimated (see Putman et al. 2002, and Fig. 1 of Schwarz \& Wakker 2004). Existing substructure in HVCs (whether imprinted during their formation or already due to the interaction with the background medium) will accelerate the disruption. The presence of substructure is equivalent to a gas volume filling factor $f_{V}<1$, i.e. some part of the cloud volume is at densities lower than the mean density (and if $f_{V} \ll 1$, a large part). Since the dynamical instabilities responsible for fragmentation have shorter growth timescales at lower density contrasts, a small volume filling factor promotes cloud disruption. Moreover, the sound speed will be larger and the cooling time will be longer in a large part of the cloud volume, resulting in a dominance of dynamical over thermal effects.

\subsubsection{Effects Increasing $\tau_{0}$ and $D_{0}$}

There are also mechanisms that will potentially increase the lifetimes of the halo clouds. Magnetic fields can suppress the dynamical instabilities (Chandrasekhar 1961), thus possibly leading to longer cloud lifetimes. Two-dimensional numerical models seem to support this notion (Konz et al. 2002; see also Palotti et al. 2008), however, the dominant instabilities allow interchange modes in three dimensions, possibly with growthrates above the hydrodynamical ones (e.g. Stone \& Gardiner 2007 for the Rayleigh-Taylor instability). Currently available three-dimensional models of HVCs including magnetic fields (Santillan et al. 2004) are limited to clouds starting at $z=2.5 \mathrm{kpc}$ above the plane and thus cannot address the question of cloud disruption over longer distances. Magnetic field estimates in HVCs are scarce - an estimate of $11.4 \pm 2.4 \mu \mathrm{G}$ in a single $\mathrm{HVC}$ (Kazès et al. 1991) could not be confirmed (Verschuur 1995: $-0.1 \pm 1.8 \mu \mathrm{G}$ for the same object). Indirect estimates based on cosmic ray confinement quote a few $\mu \mathrm{G}$ (Vallee 1990 for the Galaxy, and Beck et al. 1994 for the starburst galaxy NGC 253; see also Gaensler et al.|2008). With typical halo gas parameters, this would result in a plasma $\beta$ of order unity, i.e. the fields might not be dominant. Clearly, the effects of magnetic fields need to be explored further.

Vieser \& Hensler (2007b a argue on the basis of highresolution two-dimensional models of HVCs that heat conduction should play a substantial role in stabilizing the clouds against disruption by introducing a smooth transition layer between the hot and cold gas. Yet the effect seems to extend only the lifetime of a uniform-density cloud by a factor of two, while centrally condensed clouds (see Figs. 4a, 6a and 9a of Vieser \& Hensler 2007b) are not strongly affected, presumably because they already possess a smooth transition layer, intrinsically weakening the dynamical instabilities.

Viscosity could also play a role in stabilizing the cloud against dynamical instabilities (e.g. Chandrasekhar 1961) by introducing a finite shear layer and thus reducing the relative velocities essential for a rapid (and unconditional) growth of the Kelvin-Helmholtz instability. Since viscosity is included in our numerical scheme at (resolution-limited) Reynolds numbers smaller than those expected for the Galactic halo, its stabilizing effect is actually over-estimated in our models.

Given the distance constraints on the HVCs discussed here, our model HVCs are not massive enough to be self-gravitating, nor are they expected to be dominated by dark matter (e.g. Wakker 2004). Thus, we are neglecting the otherwise stabilizing effect of self-gravity (e.g. Murray et al. 1993) and of external potentials (e.g. Mori \& Burkert 2000).

\subsection{Consequences for Feeding the Galactic Disc}

Taking our estimated disruption timescales and distances, we find that clouds at $10 \mathrm{kpc}$ or less above the disk with $\mathrm{HI}$ masses $<10^{4.5} \mathrm{M}_{\odot}$ are highly unlikely to make it to the disk in the form of neutral hydrogen clouds. Because of the limited mass range sampled $\left(3 \times 10^{2}<M_{H I}<4.4 \times 10^{4} \mathrm{M}_{\odot}\right)$, we can only extrapolate our model data to predict the evolution of larger HVCs such as Complex $\mathrm{C}\left(\right.$ at $\approx 10^{6} \mathrm{M}_{\odot}$; see Figure 6 ). These large complexes are often seen to consist of numerous smaller clouds when observed at higher resolution (Schwarz \& Wakker 2004; Putman et al. 2002; Hsu et al. 2009), so much of their mass may also have difficulty making it to the disk in cold form.

The lack of surviving clouds has implications for the accretion of gas from satellites. The Magellanic Stream is an example of a larger complex breaking up into smaller clouds at the tip (Stanimirović et al. 2008) and via the numerous small clouds found along its length (Putman et al. 2003b). Given the distance of the Magellanic Clouds ( $\sim 60 \mathrm{kpc}$ ) the majority of the Stream is unlikely to make it to the disk in the form of cold HI clouds (see also Bland-Hawthorn et al. 2007, Bland-Hawthorn 2008). Excluding the Magellanic System, Grcevich \& Putman (2009) find that all satellites within $270 \mathrm{kpc}$ of the Milky Way or Andromeda are devoid of HI gas. This gas was most likely primarily stripped at the satellite galaxy's perigalacticon, but this still means the gas was stripped from satellites at typical distances of 20-120 kpc. Given these distances the majority of the gas stripped from satellites is unlikely to make it to the galaxy in cold form. If the fate of satellite galaxy gas is to ultimately provide the Galaxy with star formation fuel, it must first be integrated into the previously existing warm/hot halo medium.

The destruction of cold clouds in the halo results in a complex multi-phase halo of warm gas intermingled with the hot and cold gas, as also evident through the absorption line observations (Sembach et al. 2003; Ben Bekhti et al. 2008) and pulsar dispersion measurements (Gaensler et al. 2008, although given the pulsar distances, most of the material they see should be part of a thick disk component. See also 4.2.1). Even if a large fraction of the clouds do not reach the disk in the form of neutral hydrogen, they still are over-dense with respect to the background medium, and thus sink towards the plane (see also Bland-Hawthorn et al. 2007). One might speculate whether such remnants contribute to the population of warm ionized medium (WIM) clouds in the hot coronal gas (Reynolds 1993; Gaensler et al. 2008). Once they are within 1-2 kpc of the Galactic disk, the 
gas flows driven by feedback from massive star forming regions (Galactic fountain, see e.g. Bregman 2004 for a discussion) will most likely lead to compressions and recooling, eventually integrating the former HVC material into the disk as new star formation fuel. This could be easily tested by placing tracer particles in models of stratified Galactic disks including supernova feedback, such as by Joung \& Mac Low (2006).

The WIM phase is difficult to trace in our simulations, as it moves at slower velocities than the HI clouds due to being more diffuse, and thus leaves the simulation domain rather quickly (within $39 \mathrm{Myr}$ at $50 \mathrm{~km} \mathrm{~s}^{-1}$ ). Yet in models Hc1b13a and Hc1b15b (Fig. 4) we were able to follow the cloud evolution long enough to observe its nearly complete deceleration close to the plane, and the subsequent recooling of stripped material. These models suggest that warm ionized gas stripped from a HVC can recool at low $z$ (even without gas dynamics within the disk, as mentioned above), and thus re-form cold HI clouds. Because of their velocities, they would be classified as intermediate or low-velocity clouds (IVCs, LVCs). These LVCs may be similar in nature to the discrete clouds recently detected in multiple directions by several HI surveys (Lockman 2002; Stanimirović et al. 2006; Ford et al. 2008). These observed clouds are small and cold and are most likely embedded in the WIM extending $\sim 3 \mathrm{kpc}$ above the plane. If they are a mixture of accreting gas from the halo and fountain material, the LVCs should have sub-solar metallicities and may themselves show head-tail cloud structures as they sink into the Galactic disk.

\section{SUMMARY}

Motivated by the observed head-tail structure of HVCs (e.g. Brüns et al. 2000; Putman et al., in prep.; Fig. 2) and their potential to constrain the properties of the diffuse Galactic halo, we study numerically the disruption of HVCs during their passage through the Galactic halo. Our experiments address the two possible extremes of HVC trajectories, namely (a) assuming a constant background halo density and pressure, and (b) assuming an isothermal, hydrostatic halo with the cloud falling straight towards the disk. These are our findings:

Cloud Morphology and Evolution - In agreement with previous numerical studies, the clouds develop a pronounced head-tail structure due to the interaction with the background halo medium (Fig. 2). Cooling does not necessarily stabilize the cloud, especially when the dynamical timescales are shorter than the cooling timescale (Fig. 33). The velocity difference between head and tail is consistent with observed lags (Fig. 4), and we suggest that ob- served radial cloud velocities should be correlated with the shape of the cloud and the head-tail lag.

Cloud Disruption and Characteristic Distances - The limited numerical resolution and our conservative parameter choice (4.2.1) renders the estimated cloud disruption timescales and distances as upper limits. Therefore, HVCs with HI masses $<10^{4.5} \mathrm{M}_{\odot}$ will lose their HI content within $10 \mathrm{kpc}$ or less (Fig. 6). Extrapolating to more massive complexes (Fig. 7), extreme ram pressures (e.g. $n_{h}>10^{-2} \mathrm{~cm}^{-3}$ and $\left.v>100 \mathrm{~km} \mathrm{~s}^{-1}\right)$ or the fact that these complexes have abundant substructure (see 4.2.1) could lead to much of their HI mass being lost before reaching the disk. Material from the Magellanic Stream and other dwarf galaxies is not expected to survive the trip to the disk in the form of HI clouds.

The Lower Halo and Upper Disk: Fueling Star FormationWarm ionized material stripped from the HI clouds is still falling towards the disk (albeit at lower velocities). This material might constitute some of the extended layer of warm ionized gas (Reynolds 1993; Gaensler et al. 2008). Some of this material can actually recool and thus reform HI cloudlets close to the disk. Because of their low velocities, such clouds would be identified as IVCs or LVCs. We argue that the HVC remnants still could merge with the Galactic disk as WIM clouds or via recooling close to the disk, and eventually fuel the star formation in our Galaxy via a warm cosmic rain.

We are grateful to the referee, R. Benjamin, for a very detailed, critical and thorough report, and for all the prying questions by which he made us expand and strengthen our presentation considerably. Comments by J. Bullock, J. Bland-Hawthorn, T. Kaufmann and A. Maller on an earlier version of the manuscript are highly appreciated. Computations were performed at the National Center for Supercomputing Applications (AST 060034) and on the local PC cluster Star, perfectly maintained and administered by J. Hallum \& J. Billings. We acknowledge support from the Research Corporation and from NSF grants AST 0707597, AST 0748334 and AST 0807305. The manuscript was revised substantially at the Kavli Institute for Theoretical Physics (KITP) during the last week of the "Building the Galaxy"Program, supported by NSF PHY05-51164. Thanks go to J. Kollmeier and the KITP for the invitation, and to J. Peek and J. Bland-Hawthorn for discussions. This work has made use of NASA's Astrophysics Data System. Last but not least, F.H. thanks $S$. Putman for many inspirational runs.

\section{REFERENCES}

Beck, R., Carilli, C. L., Holdaway, M. A., \& Klein, U. 1994, A\&A, 292, 409

Ben Bekhti, N., Richter, P., Westmeier, T., \& Murphy, M. T. 2008, A\&A, 487, 583

Benjamin, R. A. \& Danly, L. 1997, ApJ, 481, 764

Bhatnagar, P. L., Gross, E. P., \& Krook, M. 1954, Physical Review, 94, 511

Bland-Hawthorn, J. 2008, ArXiv e-prints

Bland-Hawthorn, J., Sutherland, R., Agertz, O., \& Moore, B. 2007, ApJ, 670, L109
Bregman, J. N. 2004, in Astrophysics and Space Science Library, Vol. 312, High Velocity Clouds, ed. H. van Woerden, B. P. Wakker, U. J. Schwarz, \& K. S. de Boer, 341

Bregman, J. N. 2007, ARA\&A, 45, 221

Bregman, J. N. \& Lloyd-Davies, E. J. 2007, ApJ, 669, 990

Brüns, C., Kerp, J., Kalberla, P. M. W., \& Mebold, U. 2000, A\&A, 357, 120

Brüns, C., Kerp, J., \& Pagels, A. 2001, A\&A, 370, L26

Burkert, A. \& Lin, D. N. C. 2000, ApJ, 537, 270

Burton, W. B., Braun, R., \& Chengalur, J. N. 2001, A\&A, 369, 616 
Chandrasekhar, S. 1961, Hydrodynamic and hydromagnetic stability (International Series of Monographs on Physics, Oxford: Clarendon, 1961)

Cowie, L. L. \& McKee, C. F. 1977, ApJ, 211, 135

Danly, L., Albert, C. E., \& Kuntz, K. D. 1993, ApJ, 416, L29

Ferrière, K. M. 2001, Reviews of Modern Physics, 73, 1031

Ford, H. A., McClure-Griffiths, N. M., Lockman, F. J., Bailin, J., Calabretta, M. R., Kalberla, P. M. W., Murphy, T., \& Pisano, D. J. 2008, ApJ, 688, 290

Fraternali, F., Oosterloo, T., Sancisi, R., \& van Moorsel, G. 2001, ApJ, 562, L47

Fukugita, M. \& Peebles, P. J. E. 2006, ApJ, 639, 590

Gaensler, B. M., Madsen, G. J., Chatterjee, S., \& Mao, S. A. 2008, Publications of the Astronomical Society of Australia, 25, 184

Grcevich, J. \& Putman, M. E. 2009, ArXiv e-prints

Heitsch, F. \& Hartmann, L. 2008, ApJ, 689, 290

Heitsch, F., Hartmann, L. W., Slyz, A. D., Devriendt, J. E. G., \& Burkert, A. 2008, ApJ, 674, 316

Howk, J. C., Sembach, K. R., \& Savage, B. D. 2006, ApJ, 637, 333

Joung, M. K. R. \& Mac Low, M.-M. 2006, ApJ, 653, 1266

Kaufmann, T., Mayer, L., Wadsley, J., Stadel, J., \& Moore, B. 2006, MNRAS, 370, 1612

Kazès, I., Troland, T. H., \& Crutcher, R. M. 1991, A\&A, 245, L17

Klein, R. I., McKee, C. F., \& Colella, P. 1994, ApJ, 420, 213

Konz, C., Brüns, C., \& Birk, G. T. 2002, A\&A, 391, 713

Korycansky, D. G., Zahnle, K. J., \& Mac Low, M.-M. 2000, Icarus, 146, 387

Lockman, F. J. 2002, ApJ, 580, L47

Mac Low, M.-M. \& Zahnle, K. 1994, ApJ, 434, L33

Maller, A. H. \& Bullock, J. S. 2004, MNRAS, 355, 694

Mori, M. \& Burkert, A. 2000, ApJ, 538, 559

Murali, C. 2000, ApJ, 529, L81

Murray, S. D., White, S. D. M., Blondin, J. M., \& Lin, D. N. C. 1993, ApJ, 407, 588

Nulsen, P. E. J. 1982, MNRAS, 198, 1007

Palotti, M. L., Heitsch, F., Zweibel, E. G., \& Huang, Y.-M. 2008, ApJ, 678, 234

Peek, J. E. G., Putman, M. E., McKee, C. F., Heiles, C., \& Stanimirović, S. 2007, ApJ, 656, 907

Prendergast, K. H. \& Xu, K. 1993, J. Chem. Phys., 109, 53

Putman, M. E., Bland-Hawthorn, J., Veilleux, S., Gibson, B. K., Freeman, K. C., \& Maloney, P. R. 2003a, ApJ, 597, 948

Putman, M. E., de Heij, V., Staveley-Smith, L., Braun, R. Freeman, K. C., Gibson, B. K., Burton, W. B., Barnes, D. G., Banks, G. D., Bhathal, R., de Blok, W. J. G., Boyce, P. J., Disney, M. J., Drinkwater, M. J., Ekers, R. D., Henning, P. A. Jerjen, H., Kilborn, V. A., Knezek, P. M., Koribalski, B. Malin, D. F., Marquarding, M., Minchin, R. F., Mould, J. R., Oosterloo, T., Price, R. M., Ryder, S. D., Sadler, E. M. Stewart, I., Stootman, F., Webster, R. L., \& Wright, A. E. 2002, AJ, 123, 873

Putman, M. E., Staveley-Smith, L., Freeman, K. C., Gibson, B. K., \& Barnes, D. G. 2003b, ApJ, 586, 170

Quilis, V. \& Moore, B. 2001, ApJ, 555, L95

Reynolds, R. J. 1993, in American Institute of Physics Conference Series, Vol. 278, Back to the Galaxy, ed. S. S. Holt \& F. Verter, 156

Roulston, M. S. \& Ahrens, T. J. 1997, Icarus, 126, 138

Ruszkowski, M., Enßlin, T. A., Brüggen, M., Heinz, S., \& Pfrommer, C. 2007, MNRAS, 378, 662

Sanders, W. T., Lieu, D., McCammon, D., Rocks, L. Vaillancourt, J. E., Galeazzi, M., Figueroa-Feliciano, E., Kelley, R. L., Mushotzky, R. F., Porter, F. S., Stahle, C. K., \& Szymkowiak, A. E. 2002, in Bulletin of the American Astronomical Society, Vol. 34, Bulletin of the American Astronomical Society, 1178
Santillan, A., Franco, J., \& Kim, J. 2004, Journal of Korean Astronomical Society, 37, 233

Schwarz, U. J. \& Wakker, B. P. 2004, in Astrophysics and Space Science Library, Vol. 312, High Velocity Clouds, ed. H. van Woerden, B. P. Wakker, U. J. Schwarz, \& K. S. de Boer, 145

Sembach, K. R., Wakker, B. P., Savage, B. D., Richter, P., Meade, M., Shull, J. M., Jenkins, E. B., Sonneborn, G., \& Moos, H. W. 2003, ApJS, 146, 165

Slyz, A. \& Prendergast, K. H. 1999, A\&AS, 139, 199

Slyz, A. D., Devriendt, J. E. G., Silk, J., \& Burkert, A. 2002, MNRAS, 333, 894

Sommer-Larsen, J. 2006, ApJ, 644, L1

Stanimirović, S., Hoffman, S., Heiles, C., Douglas, K. A., Putman, M., \& Peek, J. E. G. 2008, ApJ, 680, 276

Stanimirović, S., Putman, M., Heiles, C., Peek, J. E. G., Goldsmith, P. F., Koo, B.-C., Krčo, M., Lee, J.-J., Mock, J., Muller, E., Pandian, J. D., Parsons, A., Tang, Y., \& Werthimer, D. 2006, ApJ, 653, 1210

Stone, J. M. \& Gardiner, T. 2007, ApJ, 671, 1726

Sutherland, R. S. \& Dopita, M. A. 1993, ApJS, 88, 253

Thilker, D. A., Braun, R., Walterbos, R. A. M., Corbelli, E., Lockman, F. J., Murphy, E., \& Maddalena, R. 2004, ApJ, 601, L39

Thom, C., Peek, J. E. G., Putman, M. E., Heiles, C., Peek, K. M. G., \& Wilhelm, R. 2008, ApJ, 684, 364

Thom, C., Putman, M. E., Gibson, B. K., Christlieb, N., Flynn, C., Beers, T. C., Wilhelm, R., \& Lee, Y. S. 2006, ApJ, 638, L97 Vallee, J. P. 1990, A\&A, 239, 57

van Woerden, H., Schwarz, U. J., Peletier, R. F., Wakker, B. P., \& Kalberla, P. M. W. 1999, Nature, 400, 138

Verschuur, G. L. 1995, ApJ, 451, 645

Vieser, W. \& Hensler, G. 2007a, A\&A, 475, 251

-. 2007b, A\&A, 472, 141

Vietri, M., Ferrara, A., \& Miniati, F. 1997, ApJ, 483, 262

Wakker, B. P. 2001, ApJS, 136, 463

Wakker, B. P. 2004, in Astrophysics and Space Science Library,

Vol. 312, High Velocity Clouds, ed. H. van Woerden, B. P.

Wakker, U. J. Schwarz, \& K. S. de Boer, 25

Wakker, B. P. \& van Woerden, H. 1991, A\&A, 250, 509

-. 1997, ARA\&A, 35, 217

Wakker, B. P., York, D. G., Howk, J. C., Barentine, J. C. Wilhelm, R., Peletier, R. F., van Woerden, H., Beers, T. C. Ivezić, Ž., Richter, P., \& Schwarz, U. J. 2007, ApJ, 670, L113

Wakker, B. P., York, D. G., Wilhelm, R., Barentine, J. C., Richter, P., Beers, T. C., Ivezić, Ž., \& Howk, J. C. 2008, ApJ, 672,298

Westmeier, T., Brüns, C., \& Kerp, J. 2005, A\&A, 432, 937

White, S. D. M. \& Frenk, C. S. 1991, ApJ, 379, 52

White, S. D. M. \& Rees, M. J. 1978, MNRAS, 183, 341

Wolfire, M. G., Hollenbach, D., McKee, C. F., Tielens,

A. G. G. M., \& Bakes, E. L. O. 1995a, ApJ, 443, 152

Wolfire, M. G., McKee, C. F., Hollenbach, D., \& Tielens, A. G. G. M. 1995b, ApJ, 453, 673

$\mathrm{Xu}$, K. 2001, Journal of Computational Physics, 171, 289 